\section{Efficacy of Beauveria bassiana on Colonized Millet Seed as a Biopesticide for the Control of Shore Flies}

\author{
M.E. Stanghellini and Z.A. El-Hamalawi \\ Department of Plant Pathology, University of California, Riverside, CA 92521
}

Additional index words: Scatella stagnalis, shore flies, insect biocontrol, insect vectors, soilborne plant pathogens, root-infecting fungi, entomopathogenic fungus

\begin{abstract}
Shore flies (Scatella stagnalis) have been implicated as an aerial vector of several soilborne plant pathogens of greenhouse crops. A strain of Beauveria bassiana $(B b)$, isolated from a shore fly cadaver, was evaluated as a biopesticide for the control of shore flies. $B b$ was grown on autoclaved millet seed for 2 weeks, air-dried at $24^{\circ} \mathrm{C}$ for 7 days and stored in paper bags. Dried $B b$-colonized millet seeds were broadcast on the surface of pots containing potting medium naturally-infested with larvae and pupae or pots infested only with adult flies. Controls consisted of insect-infested pots amended with autoclaved millet seeds. Pots of the same treatment were placed in insect-proof screen cages. At daily intervals for 15 days, adult fly populations were recorded in each cage. In cages initially containing only larvae and pupae, adult fly populations in the control (no $B b$ ) treatment reached a final population of 70 adults on day 15 . In contrast only two adult flies were observed in the $B \boldsymbol{b}$-treatment. In cages containing only adult shore flies, the entire population was dead within 10 to 12 days in the $B b$-treatment. In the absence of $B b, 65 \%$ of the initial population of adults was still alive on day 12 .
\end{abstract}

Greenhouse floricultural, ornamental, transplant and vegetable crops are subject to numerous biotic diseases, which place major economic constraints upon their production and/or marketability. Management of these diseases is based on preventive as well as curative disease control strategies. Preventive management strategies, i.e., the exclusion of the pathogen from the production area, are more desirable for a number of economic and ecological reasons. However, exclusion is dependent upon knowledge of the source(s) of pathogen introduction and/or spread within a commercial production facility. This is particularly true in the case of soilborne, root-infecting fungal pathogens. Documented sources of pathogen introduction and/or spread include pathogeninfested/infected seed or seedlings, potting media, air, and irrigation water (Jarvis, 1992; Stanghellini and Rasmussen, 1994).

Recently, several dipterous insects [i.e., adult shore flies (Scatella stagnalis Fallen), fungus gnats (Bradysia spp.) and moth flies (Psychoda spp.)], which historically have been regarded primarily as a nuisance (i.e., deposition of excrement on foliage by shore flies or damage to roots from the feeding of fungus gnat larvae), have been implicated as aerial vectors of some soilborne fungal pathogens (El-Hamalawi and Stanghellini, 2004 and 2005; Gillespie and Menzies, 1993; Goldberg and Stanghellini, 1990; Kalb and Miller, 1986; Stanghellini et al., 1999), particularly those that sporulate above-ground on infected stem

Received for publication 15 Dec. 2004. Accepted for publication $24 \mathrm{Feb} .2005$. This research was partially supported by grants from the USDA-ARS Floral and Nursery Research Initiative. We thank Denny J. Bruck for his assistance in obtaining the ITS sequencing of Beauveria bassiana, strain UCR-03.

${ }^{1}$ To whom reprint requests should be addressed; e-mailmichael.stanghellini@ucr.edu. the control of fungus gnats but few provide consistent or efficacious control of shore flies. During the course of our investigations on the role of shore flies as vectors of soilborne plant pathogenic fungi, we encountered and isolated an entomopathogenic fungus from shore fly cadavers following an epizootic event (Fig. 1). A single spore culture of the fungus, herein designated as strain UCR-03, was identified morphologically (and verified by ITS sequencing, D.J. Bruck, personal communication) as Beauveria bassiana (Balsamo) Vuillemin.

Beauveria bassiana $(B b)$ is a naturally occurring, soilborne entomopathogenic fungus (Deuteromycetes) that occurs world wide. It attacks a wide range of immature and adult insects and there are numerous strains of this fungus which exhibit considerable variation in pathogenicity, virulence and host range. The fungus produces conidia which, upon contact with the insect, germinate and penetrate the host directly through the exoskeleton. Subsequent hyphal colonization of internal host tissues, coupled with the production of toxins, results in death of the insect, followed by extensive external pathogen sporulation on the insect cadaver (Ferron, 1978).

Our preliminary studies showed that adult shore flies were attracted to sporulating $B b$ colonies. This latter observation (which paralleled our observations of insect attraction to plant pathogenic fungi sporulating on the surface of infected host tissues, El-Hamalawi and Stanghellini, 2004 and 2005) led, as reported herein, to the evaluation and subsequent formulation of $B$. bassiana on colonized millet seed as biopesticidal bait for the control of shore flies. A preliminary report has been published (Stanghellini and El-Hamalawi, 2004).

\section{Materials and Methods}

A stock culture of $B$. bassiana (Bb), strain UCR-03, was maintained on potato dextrose agar (PDA). A colony of shore flies was established and maintained on algal-infested 
rockwool blocks in a growth chamber as previously described (El-Hamalawi and Stanghellini, 2005).

Pathogenicity studies. Pathogenicity of strain UCR-03 to shore fly adults and larvae was evaluated as follows: 30 adult shore flies were collected by aspiration from the colony and placed in a 2-L plastic canister containing a 7-d-old sporulating PDA petri dish (9-cmdiameter) culture of $B$. bassiana. Controls consisted of 30 adult shore flies placed in separate canisters containing either a petri dish containing sterile PDA or a 7-d-old sporulating PDA petri dish culture of Penicillium sp. Penicillium was used as an irritant control because it produces abundant conidia of a size similar to $B b$. Adult flies placed in canisters with either $B b$ or Penicillium were, within $2 \mathrm{~h}$, coated with conidia of each fungus.

A similar experimental design was used to evaluate the pathogenicity of the entomopathogen to larvae of the shore fly. Fifteen larvae (third and fourth instars) were collected from the colony and placed in petri dishes (PDA) containing a 7-d-old sporulating colony of $B$. bassiana, Penicillium sp., or sterile PDA.

All treatments, which were replicated three times, were incubated at $24^{\circ} \mathrm{C}$ on the laboratory bench. Mortality data were recorded every 2 to $4 \mathrm{~h}$ for $36 \mathrm{~h}$ for studies with adult shore flies and daily for $10 \mathrm{~d}$ with shore fly larvae. Five dead adults and larvae from each treatment were randomly collected and plated onto water agar to stimulate fungal sporulation and identification of $B b$ as the probable cause of death. All experiments were repeated once.

Formulation of strain UCR-03 as biopesticidal bait. $B b$ was grown on moist autoclaved millet seed (hulled pear millet, Pennisetum glaucum L., Arrowhead Mills) for 2 weeks at $24^{\circ} \mathrm{C}$. Bb-colonized seeds were then allowed to air-dry at room temperature $\left(24^{\circ} \mathrm{C}\right)$ for $7 \mathrm{~d}$ and then stored in paper bags at $5{ }^{\circ} \mathrm{C}$ and 24 ${ }^{\circ} \mathrm{C}$ until used. Preliminary studies showed that after placement of the dried $B b$-colonized millet seed on a moist surface (i. e., rehydration), the fungus produced hyphae and then sporulated profusely on the millet seed. The shelf life of dried $B b$-colonized seed was assessed monthly over a 12 -month period as follows: $20 \mathrm{Bb}$-colonized dry millet seed from each storage temperature were plated, in triplicate, onto water agar contained in petri dishes and incubated at $24{ }^{\circ} \mathrm{C}$. At daily intervals for 6 $\mathrm{d}$, data were recorded on the percentage of dried millet seeds that supported a sporulating colony of the $B b$. Additionally, the population of $B b$ conidia on the dried colonized seeds before rehydration and at daily intervals over a 5-d period subsequent to rehydration was assessed as follows: one colonized seed was placed in a centrifuge tube containing $2 \mathrm{~mL}$ of sterile distilled water and agitated for 2 $\mathrm{min}$ in a vortex mixer. The number of conidia in the suspension was then estimated with a hemacytometer. Results were reported as the number of conidia per seed. Additionally, $0.1-\mathrm{mL}$ aliquots of the conidial suspension from sporulating seeds were dispensed onto the surface of water agar contained in petri dishes. After $18 \mathrm{~h}$ incubation at $24^{\circ} \mathrm{C}$, percent conidial germination was observed microscopically $(400 \mathrm{X})$ and recorded. The viability of conidia on the $B b$-colonized millet seed before rehydration was not determined.

Bait efficacy studies. To assess the efficacy of the $B b$-colonized millet seed as biopesticidal bait, the following experiments were conducted. Five $B b$-colonized millet seeds that had been stored at $5{ }^{\circ} \mathrm{C}$ were placed on the surface of water agar contained in a 6$\mathrm{cm}$-diameter petri dish. The latter petri dish, and a petri dish containing water agar seeded with 5 autoclaved millet seeds, were both placed in a 2-L clear plastic canister with 10 adult shore flies. The control consisted of a 2-L canister with 10 adult shore flies and a petri dish containing water agar seeded with 5 autoclaved millet seeds. All treatments were incubated at $24^{\circ} \mathrm{C}$ on the laboratory bench. Mortality was recorded daily for $14 \mathrm{~d}$. There were three replications of each treatment and the experiment was repeated once.

Greenhouse studies. Dried Bb-colonized millet seeds were broadcast (20 seeds/pot)

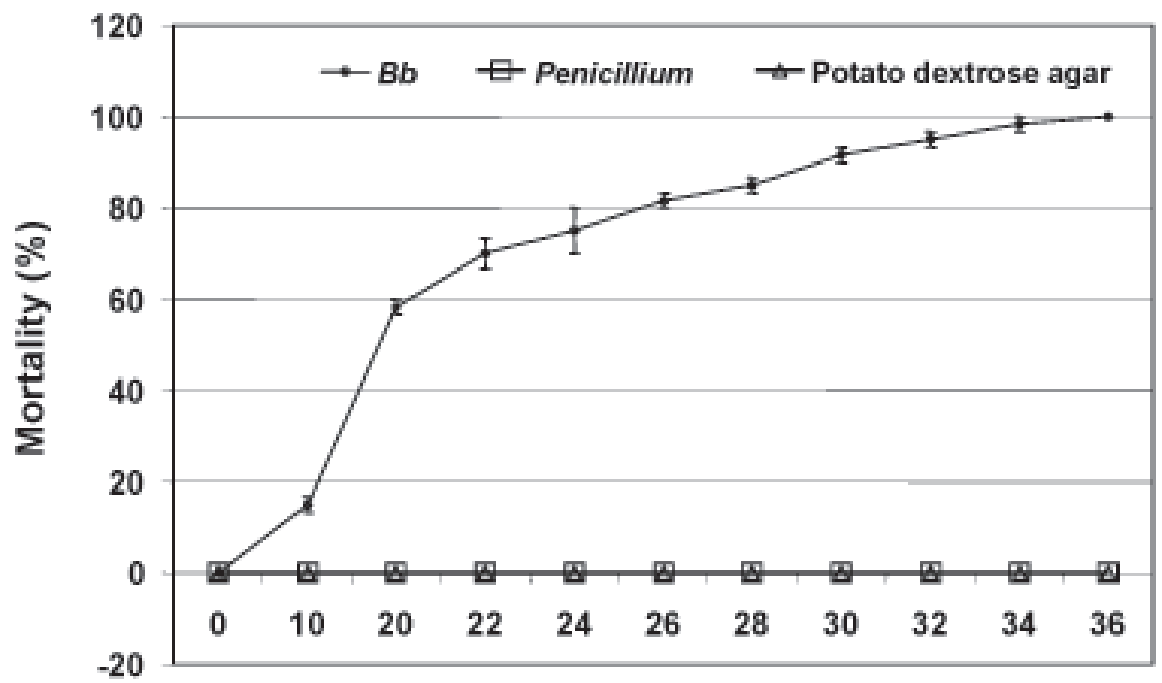

Time (hours)

Fig. 2. Mortality of adult shore flies following exposure to an entomopathogenic fungus, (Beauveria bassiana, $B b$ ), a saprophytic fungus (Penicillium sp.), and a nutrient substrate (potato dextrose agar). Assessments were made every 2 to $4 \mathrm{~h}$ for $36 \mathrm{~h}$ after caging 30 adult shore flies in each of three separate canisters containing one of the two fungi or the nutrient substrate. Values represent the mean and standard error of combined data from two separate experiments.

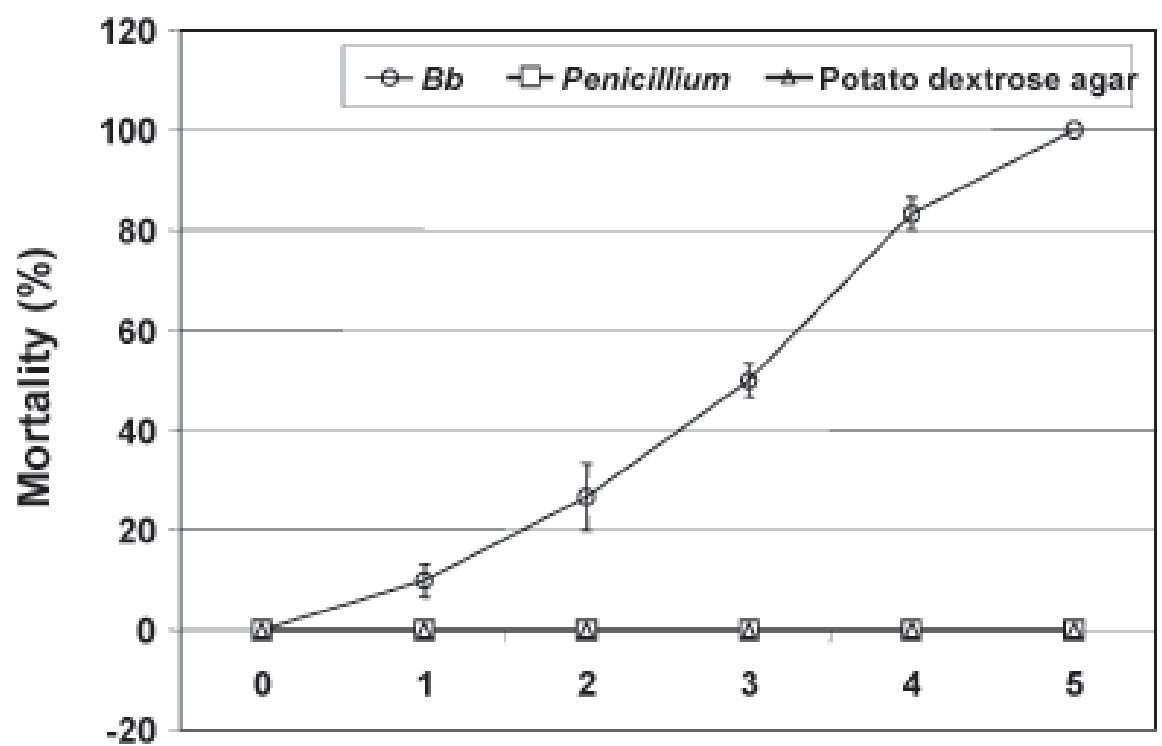

\section{Time (days)}

Fig. 3. Mortality of shore fly larvae following exposure to an entomopathogenic fungus, (Beauveria bassiana, $B$ ), a saprophytic fungus (Penicillium sp.), and a nutrient substrate (potato dextrose agar). Assessments were made daily for $5 \mathrm{~d}$ after caging 15 shore fly larvae in separate canisters containing one of the two fungi or the nutrient substrate. Values represent the mean and standard error of combined data from two separate experiments. 
on the surface of five, 15 -cm-diameter pots containing potting medium naturally infested with algae, larvae and pupae of shoreflies (all adults were removed) or algae-infested pots that were not infested with shore flies. Pots of the same treatment were then placed in separate insect-proof screen cages $(50 \mathrm{~cm}$ in length, $40 \mathrm{~cm}$ in width, and $50 \mathrm{~cm}$ in height) on benches in the greenhouse (ambient temperatures ranged from 20 to $28{ }^{\circ} \mathrm{C}$ daily). Immediately after placement of the cages collected from the colony and released into those screen cages containing the pots that were infested only with algae and seeded with in the greenhouse, 25 adult shore flies were

the dried $B b$-colonized millet seeds. Controls consisted of the same treatments amended with autoclaved millet seeds in separate screen cages. Adult fly populations in all treatments, as well as the number of $B b$-colonized millet seeds that supported sporulating colonies of the entomopathogen, were determined visually and recorded daily for $15 \mathrm{~d}$. The experiments were repeated once.

\section{Results.}

Pathogenicity studies. Within $2 \mathrm{~h}$ after placement in canisters containing either $B b$ or Penicillium sp., all adult shore flies were

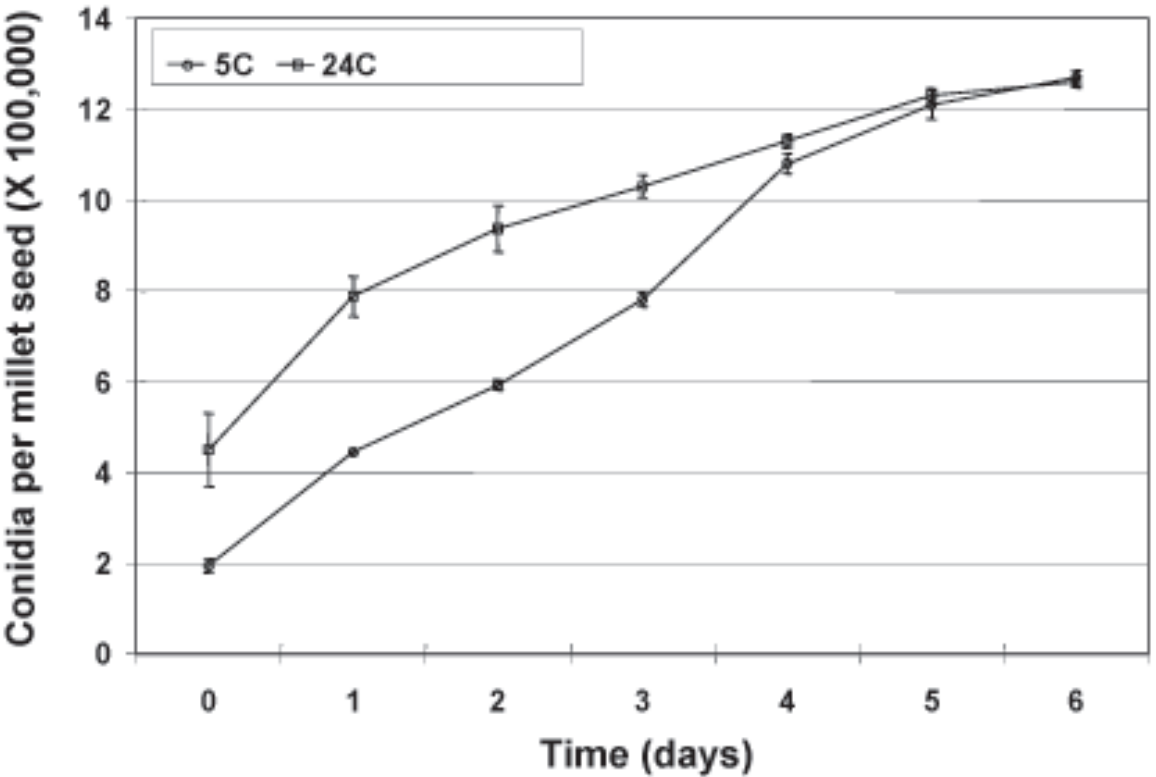

Fig. 4. Progression of sporulation over time of Beauveria bassiana on dried colonized millet (stored at 5 or $24^{\circ} \mathrm{C}$ ) seed following rehydration and incubation at $24^{\circ} \mathrm{C}$. Values represent the mean and standard error of two replications.

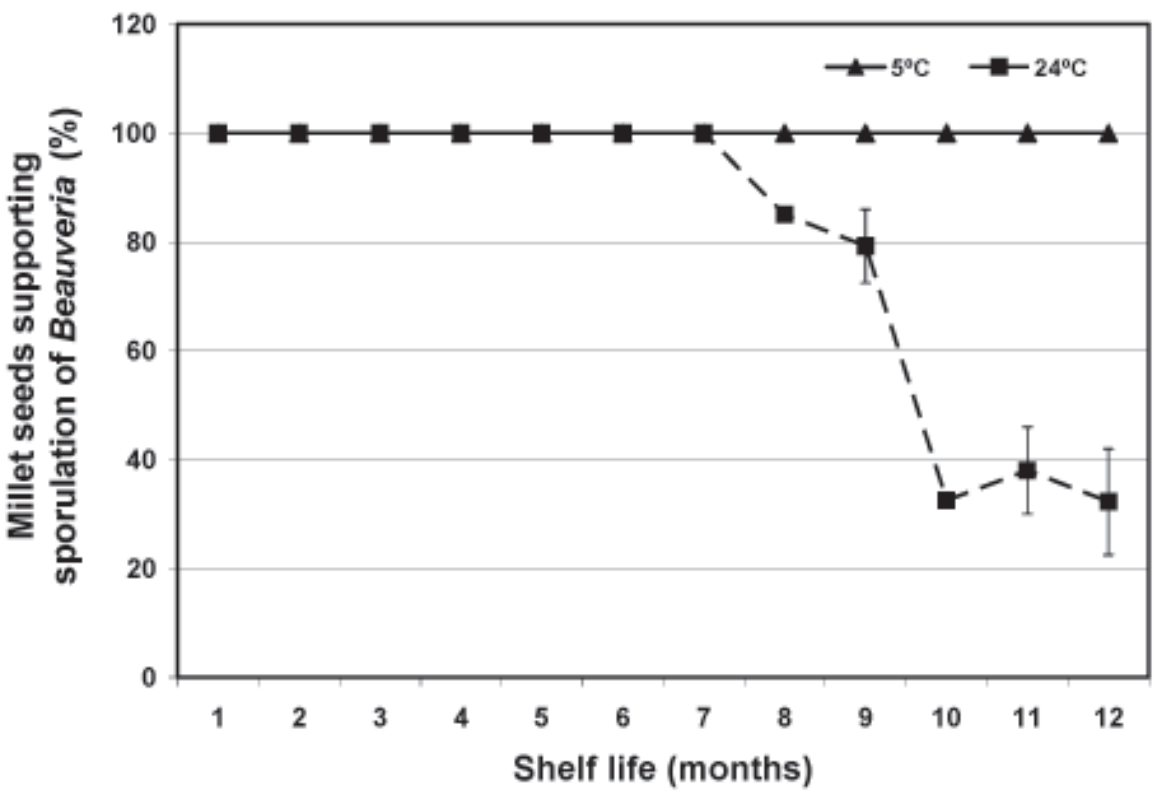

Fig. 5. Shelf life of dried Beauveria bassiana- colonized millet seed stored at 5 and $24^{\circ} \mathrm{C}$ in paper bags. Data are the percentage of dried millet seed capable of supporting sporulation of the entomopathogen upon rehydration and incubation at $24^{\circ} \mathrm{C}$. Three replications of 20 seeds each were assayed per sampling date. Values represent the mean and standard error of three replications. coated externally with conidia of each fungus. All adult shore flies were killed within $36 \mathrm{~h}$ in canisters containing $\mathrm{Bb}$, but no mortality was recorded in canisters containing Penicillium sp. or the PDA control treatments (Fig. 2). Sporulating colonies of $B b$ were observed visually on all adult cadavers incubated on water agar. All larvae were killed within $5 \mathrm{~d}$ after placement on PDA cultures of $B b$ but no mortality occurred in populations of larvae exposed to either Penicillium sp. or PDA(Fig. 3). Beauveria bassiana was observed visually on all larval cadavers incubated on water agar. Although there was no mortality of larvae in either of the two control treatments, about $70 \%$ of the larvae in the Penicillium sp. treatment had pupated by day 5 and the remaining 30\% were still feeding even after an additional incubation period of $5 \mathrm{~d}$ at which time the study was terminated. These results are consistent with our previous findings (Stanghellini and El-Hamalawi, unpublished) that shore flies can complete their entire life cycle solely on a diet of various fungi, including Penicillium $\mathrm{sp}$. In contrast, none of the larvae in the PDA treatment pupated and they all died by day 10 . However, B. bassiana was not associated with any of the latter cadavers. They apparently died of starvation.

Bait formulation. When placed on the surface of water agar in a petri dish, the $\mathrm{Bb}$ colonized millet seed that had been air-dried and stored at 5 or $24{ }^{\circ} \mathrm{C}$ for various periods of time, rapidly imbibed moisture. Hyphae of $B b$ were visually evident on the surface of the seed after $12 \mathrm{~h}$ and the fungus sporulated profusely on the surface of the rehydrated colonized millet seed over the next $6 \mathrm{~d}$. Populations of conidia increased from about $4.5 \times 10^{5} /$ dry seed to $1.3 \times 10^{6} /$ seed after $6 \mathrm{~d}$ incubation on water agar plates at $24{ }^{\circ} \mathrm{C}$ (Fig. 4). The percentage of dried $\mathrm{Bb}$-colonized millet seed capable of supporting growth and sporulation of $B b$ upon rehydration varied depending upon the storage temperature (Fig. 5). At $24^{\circ} \mathrm{C}$, the percentage viability remained high $(100 \%)$ for about 6 months but then declined progressively to about $20 \%$ over the next 6 months. At $5{ }^{\circ} \mathrm{C}$, the percentage viability remained at $100 \%$ over the duration (12 months) of the study. The percentage germination of conidia produced on sporulating millet seeds ranged from $96 \%$ to $100 \%$.

Bait efficacy as a pesticide. In repeated experiments, all adult shore flies were killed within $6 \mathrm{~d}$ in canisters containing petri dishes seeded with $B b$-colonized millet seed (Fig. 6) and the fungus was visually observed on all cadavers. In contrast, about $50 \%$ of the adult shore flies in the control treatment died over the duration of the experiment (14 d) and the entomopathogenic fungus was not associated with any of those cadavers.

Greenhouse studies. Within $24 \mathrm{~h}$ after seeding the surface of pots with the $B b$-colonized bait, fungal hyphae were visually observed on the surface of the individual millet seeds. All seeds supported extensive hyphal growth and sporulation over the next 4 to $5 \mathrm{~d}$ and then sporulation progressively declined over the next 8 to 9 d (Fig. 7). 
Pots infested with larvae and pupae. In pots infested only with larvae and pupae, adult fly populations in the control (no $B b$ ) treatment were first recorded on day 3 , increased progressively over the duration of the study, and reached a final population of 70 adults on day 15 (Fig. 8). In contrast, one adult fly was first observed in the $B b$ treatment on day 8 , variable populations ( 0 to 2 adults) occurred between days 9 to 14 , and a final population of 2 adults was observed on day 15. Similar results were recorded in a repeat experiment.

Pots infested with adult insects. In pots infested with adult shore flies, there was a slow progressive decline in the adult fly population over time in the control (no $\mathrm{Bb}$ ) treatment. However, about $65 \%$ of the adult population was alive after 13 days. In contrast, there was a rapid decline in the population of adult shore flies subsequent to application of $B b$-colonized millet seed and none were alive after 10 to 12 days (Fig. 9). Similar results were recorded in

\section{Discussion}

Our results demonstrate the potential efficacy of $B$. bassiana on colonized millet seed as a biopesticide for the control of shore flies. Strain UCR-03 was pathogenic to larval and adult life stages of shore flies in both laboratory and greenhouse trials. Highest and most rapid mortality rates ( $100 \%$ after $36 \mathrm{~h}$ ) occurred when adults were caged with sporulating PDA cultures of $B b$. As previously mentioned, adults in the latter experiments were visually coated with conidia within the first $2 \mathrm{~h}$ of caging. A spore coating, however, also occurred on adults caged with the Penicillium $s p$. but no mortality occurred in that treatment, which was included in the experimental design to eliminate irritation as a factor associated with mortality. Although such high levels of infestation with conidia are not realistic, they demonstrated that the strain we employed was pathogenic. Larvae appeared to be more insensitive to the entomopathogen than adults but they all died within $5 \mathrm{~d}$ after placement in petri dishes containing a sporulating colony of $B b$. In subsequent laboratory experiments employing millet seeds colonized by $B b, 100 \%$ mortality (caused by the fungus) of adults occurred after $5 \mathrm{~d}$, whereas only $50 \%$ mortality (natural death) occurred in the control treatment after $14 \mathrm{~d}$. The different mortality rates of adult shore flies exposed to $B b$ originating from PDA petri plates versus colonized millet seed most likely reflects the vastly different levels of inoculum acquired by the adults from the different inoculum sources.

Results from greenhouse experiments confirmed laboratory studies. Dried $B b$-colonized millet seeds, which were broadcast on the surface of pots containing potting medium naturally infested with larvae and pupae or potting media infested only with adult flies, imbibed moisture and the fungus sporulated continuously on the colonized bait for about $12 \mathrm{~d}$, thus providing a continuous source of conidia for infection of shore flies. In pots initially containing only larvae and pupae, adult a repeat experiment. fly populations in the control (no $B b$ ) treatment increased progressively over the duration of the study and reached a final population of 70 adults on day 15 . In contrast only two adult flies were observed in the $B b$-treatment on day 15 . These results indicated that larvae, or the immature adults emerging from existing pupae, were susceptible to $B b$. In pots containing only adult shore flies, the population of adults in the $B b$-treatment declined rapidly and the entire population was dead within 10 to $12 \mathrm{~d}$. In the absence of the pathogen, $65 \%$ of the population of adults was alive in that same time frame.

There are several commercial biological products (Botanigard 22WP, Botanigard ES, Mycotrol O, Mycotrol, and Naturalis TNO) containing patented strains of $B b$ for the control of insects in greenhouses but only Natural-

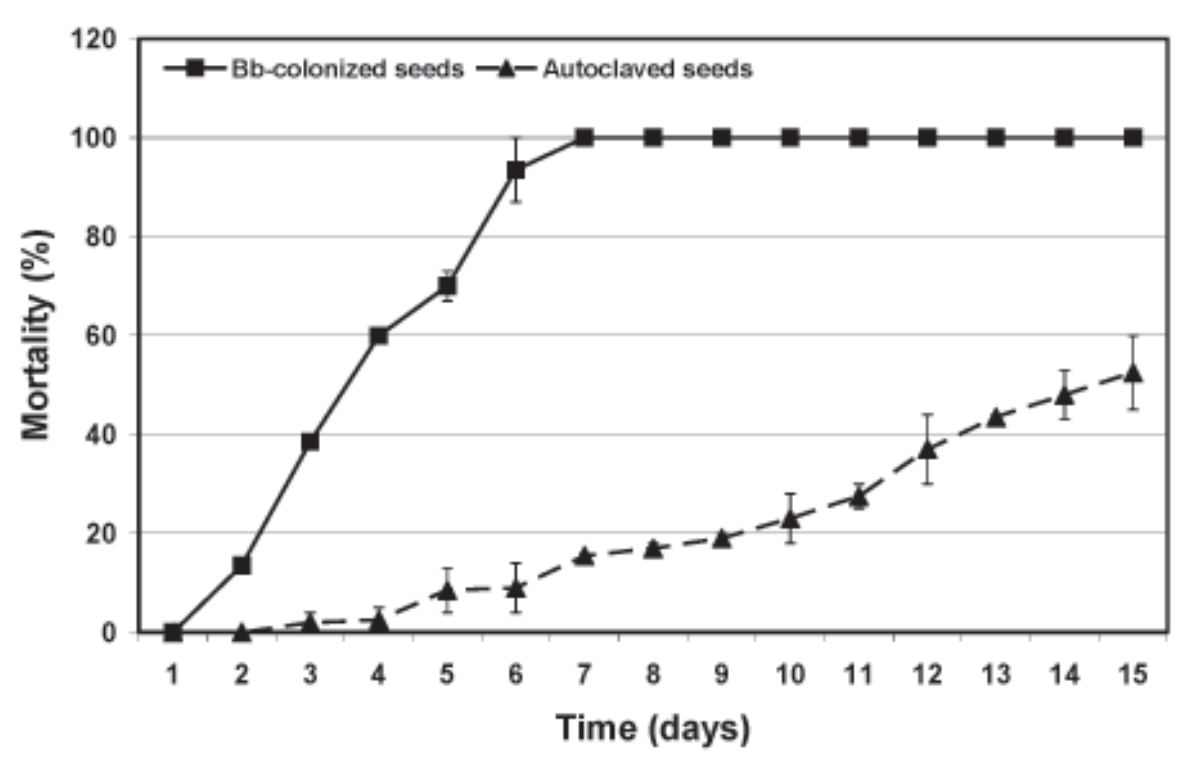

Fig. 6. Mortality of adult shore flies following exposure to Beauveria bassiana-colonized millet seeds (bait) versus autoclaved millet seeds. Values represent the mean and standard error of combined data from two separate experiments.

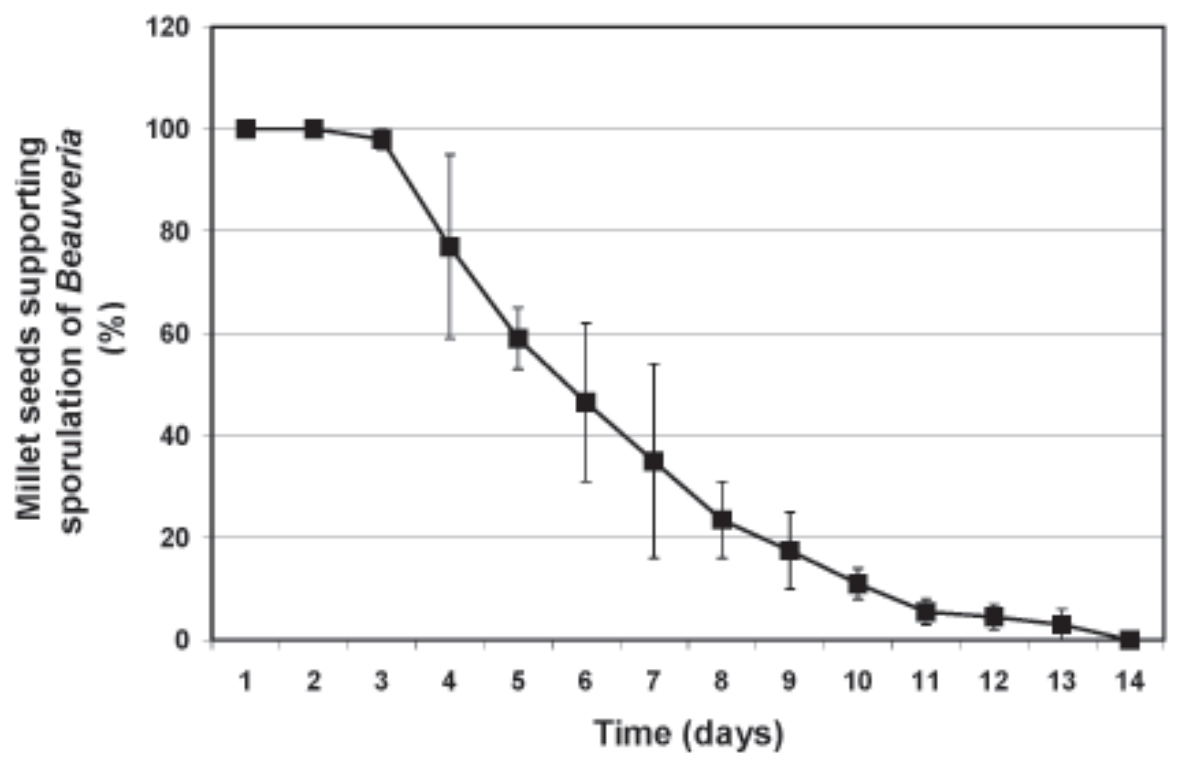

Fig. 7. The duration of sporulation by Beauveria bassiana on colonized millet seed subsequent to application of the bait to an insect-infested site. Values represent the mean and standard error of combined data from two separate experiments.

isTNO is registered for control of shore flies. All of the commercial products consist of a powder or liquid formulation containing high concentrations of conidia of the fungus (produced by proprietary fermentation processes) which are diluted in water and then applied with standard spray equipment to plant foliage, a dip Conidia must contact the insect directly to be effective and they rapidly lose their viability, thus necessitating frequent applications. In contrast, using our approach, the target pest acquires sufficient inoculum of the entomopathogen directly from the $B b$-colonized
millet seed, which then results in significant mortality. Additionally, formulation of the entomopathogen as a biopesticidal bait has several other advantages over spray applicasoil application in landscapes and containers. 


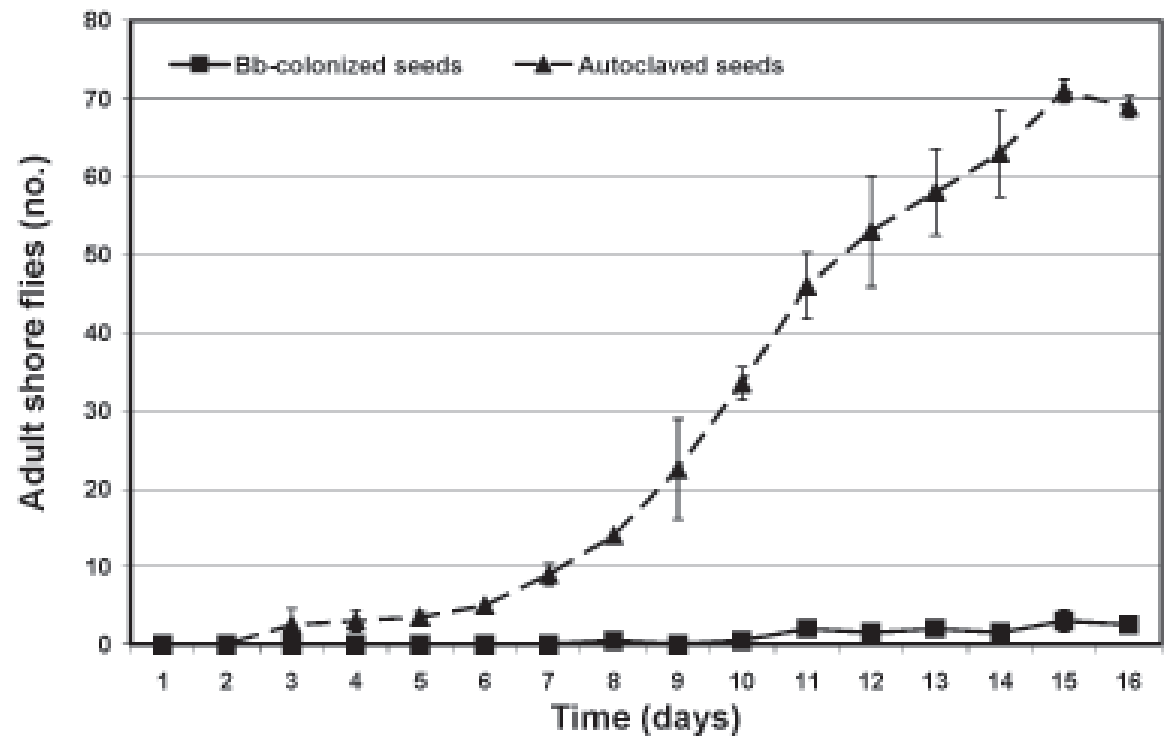

Fig. 8. Effect of amending a shore fly larva and pupa-infested potting media with Beauveria bassiana-colonized millet seed or autoclaved millet seed on the developing adult fly population under greenhouse conditions. Values represent the mean and standard error of combined data from two separate experiments.

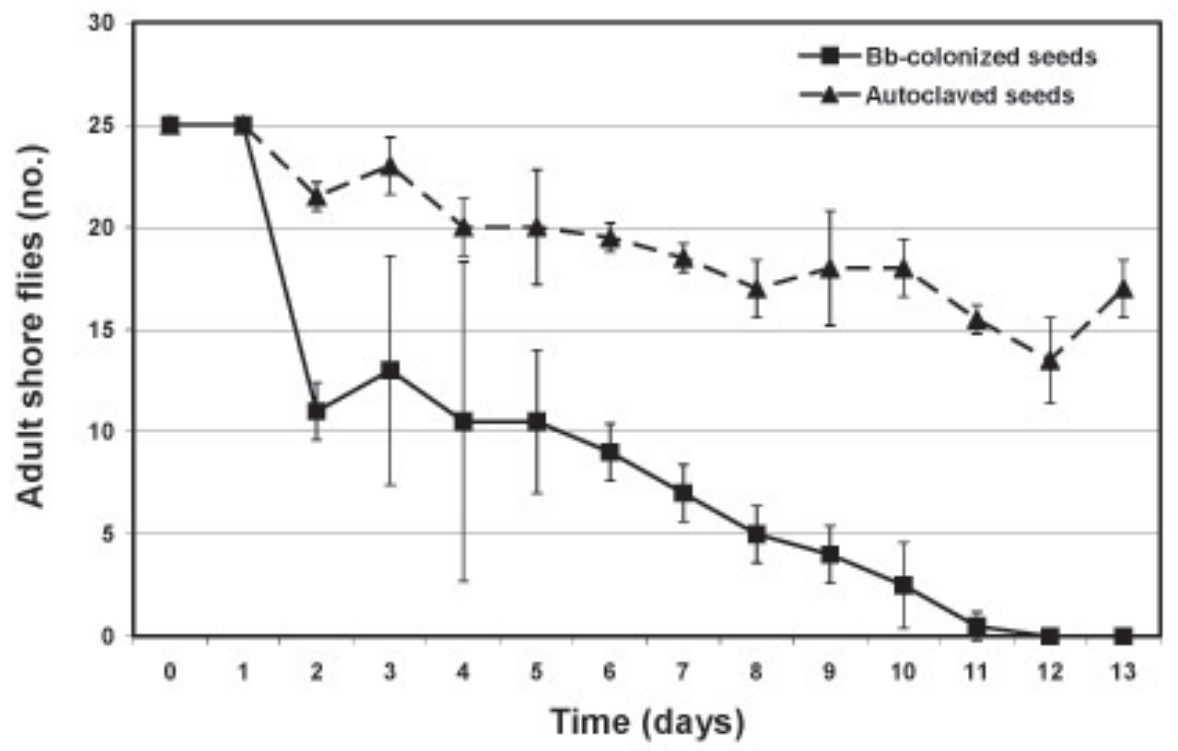

Fig. 9. Effect of amending an adult shore fly-infested potting medium with Beauveria-bassiana-colonized millet seed or autoclaved millet seed on the existing adult fly population under greenhouse conditions. Values represent the mean and standard error of combined data from two separate experiments.

tions of the entomopathogen. First, production of inoculum on millet seed is relatively simple, and does not require expensive fermentation equipment. Second, the bait formulation has a long shelf life at room (about 6 months) or refrigeration temperatures (1 year). Third, ap- decrease the application frequency associated with spray applications of conidia.

\section{Literature Cited}

El-Hamalawi, Z.A. and M.E. Stanghellini. 2004. Acquisition and aerial dissemination of Fusarium and Verticillium by adult shoreflies. Phytopathology 94:S151 (abstr.).

El-Hamalawi, Z.A. and M.E. Stanghellini. 2005. Disease development on lisianthus (Eustoma grandiflorum) following aerial transmission of Fusarium avenaceum by adult shore flies, fungus gnats, and moth flies. Plant Dis. 89:619-623.

Ferron, P. 1978. Biological control of insect pests by entomogenous fungi. Annu. Rev. Entomol. 23:409-442.

Gamliel, A., J. Katan, H. Yunis, and T. Katan. 1996. Fusarium wilt and crown rot of sweet basil: involvement of soilborne and airborne inoculum. Phytopathology 86:56-62.

Gillespie, D.R. and J.G. Menzies. 1993. Fungus gnats vector Fusarium oxysporum f.sp. radicis-lycopersici. Ann. Appl. Biol. 123:539-544.

Goldberg, N.P. and M.E. Stanghellini. 1990 Ingestion-egestion and aerial transmission of Pythium aphanidermatum by shore flies (Ephydrinae: Scatella stagnalis). Phytopathology 80:1244-1246.

Jarvis, W.R. 1992. Managing Diseases in Greenhouse Crops. APS Press, 288 pages.

Kalb, D.W. and R.L. Millar. 1986. Dispersal of Verticillium albo-atrum by fungus gnat (Bradysia impatiens). Plant Dis. 70:752-753.

Katan, T., E. Shelvin, and J. Katan. 1997. Sporulation of Fusarium oxysporum f.sp. lycopersici on stem surface of tomato plants and aerial dissemination of inoculum. Phytopathology 87:712-719.

Punja, S.K. and M. Parker. 2000. Development of Fusarium root and stem rot, a new disease on greenhouse cucumber in British Columbia, caused by Fusarium oxysporum f.sp. radicis-cucumerinum. Can. J. Plant Pathol. 22:349-363.

Rekah, Y., D. Shtienberg, and J. Katan, J. 2000. Disease development following infection of tomato and basil foliage by airborne conidia of the soilborne pathogens Fusarium oxysporum $\mathrm{f}$. sp. radicis-lycopersici and $F$. oxysporum $\mathrm{f}$. sp. basilici. Phytopathology 90:1322-1329.

Stanghellini, M.E. and S.L. Rasmussen. 1994. Hydroponics: A solution for zoosporic pathogens. Plant Dis. 78:1129-1138.

Stanghellini, M.E., S.L. Rasmussen, and D.H. Kim. 1999. Aerial transmission of Thielaviopsis basicola, a pathogen of corn-salad, by adult shore flies. Phytopathology 89:476-479.

Stanghellini, M.E. and Z.A. El-Hamalawi. 2004 Efficacy of Beauveria bassiana on colonized millet seed as a biopesticide for the control of shore flies in greenhouses. Phytopathology 94 : S98 (abstr.)

Vakalounakis, D.J. 1996. Root and stem rot of cucumber caused by Fusarium oxysporum f.sp. radicis-cucumerinum f.sp. nov. Plant Dis. 80:313-316. 